\title{
Un cadre associatif pour l'innovation technique dans les campagnes bretonnes du XXe siècle
}

L'acquisition d'une étuveuse à pommes de terre par la CUMA de SaintBrice-en-Coglès

\section{Martine Cocaud}

\section{(2) OpenEdition}

Journals

Édition électronique

URL : http://journals.openedition.org/abpo/1701

DOI : 10.4000/abpo.1701

ISBN : 978-2-7535-1483-6

ISSN : $2108-6443$

\section{Éditeur}

Presses universitaires de Rennes

\section{Édition imprimée}

Date de publication : 20 septembre 2001

Pagination : 135-145

ISBN : 978-2-86847-625-8

ISSN : 0399-0826

\section{Référence électronique}

Martine Cocaud, « Un cadre associatif pour l'innovation technique dans les campagnes bretonnes du XXe siècle ", Annales de Bretagne et des Pays de l'Ouest [En ligne], 108-3 | 2001, mis en ligne le 20 septembre 2003, consulté le 22 avril 2019. URL : http://journals.openedition.org/abpo/1701 ; DOI : 10.4000/abpo. 1701 


\title{
Un cadre associatif pour l'innovation technique dans les campagnes bretonnes du $\mathrm{XX}^{\mathrm{e}}$ siècle :
}

\author{
L'acquisition d'une étuveuse à pommes de terre \\ par la CumA de Saint-Brice-en-Coglès
}

\author{
Martine COCAUD \\ Maître de conférences en histoire contemporaine \\ CRHISCO, Université Rennes 2
}

L'adoption, par les plus nombreux, de techniques agricoles jusqu'alors réservées à quelques aventuriers fut souvent l'œuvre d'organisations agricoles locales : c'est, par exemple, par l'intermédiaire des comices cantonaux que la charrue Dombasle ou les nouvelles races de bestiaux s'imposent à la fin du xIX ${ }^{e}$ siècle. Dans l'Ouest, en 1945 lorsque la mécanisation paraît inéluctable, les CumA, organisées au niveau communal ou cantonal, vont faciliter l'adoption des tracteurs, des moissonneuses-batteuses et de bien d'autres matériels. C'est une partie de cette histoire, somme toute récente, que je souhaite évoquer dans cet article. En 1955, une Cuma est fondée à Saint-Brice en Coglès (commune du nord-est de l'llle-et-Vilaine), à l'occasion de l'achat d'une étuveuse à patates. La cohésion des agriculteurs autour de cet objet a permis la formation de plusieurs mouvements associatifs qui sont actuellement très actifs. Cet épisode du développement agricole de la Bretagne constitue une occasion de s'interroger sur certains aspects de la modernisation de l'agriculture bretonne après la Seconde Guerre mondiale.

\section{L'étuveuse à patates}

Cette machine, dont le nom sonne maintenant de façon désuète ${ }^{1}$, permet la cuisson, à fins de conservation, des pommes de terre qui craignent le gel ou qui germent trop vite ${ }^{2}$. Depuis le milieu du xixe siècle, les pommes

1. L'étuveuse revient d'actualité avec les bio-technologies : un brevet d'étuveuse à pommes de terre a été déposé en 1991 au Québec.

2. L'article intitulé " l'ensilage " extrait de La Revue de l'élevage d'août 1950, rappelle qu'autrefois on ne pouvait conserver que $70 \%$ des pommes de terre, et que le seul 
de terre étaient cultivées dans les fermes d'Ille-et-Vilaine afin de nourrir les cochons : les femmes cuisaient 3 à 4 fois par semaine la nourriture nécessaire à l'engraissement des 2 ou 3 porcs entretenus par la famille. Dans les régions très productrices (l'Allemagne par exemple), dès l'entre-deux-guerres et peut-être même avant, cette tradition a été mise à mal par l'étuveuse à patates; juste après la guerre, les agronomes français tentent de la faire connaître à ceux qui cultivent la pomme de terre pour nourrir les bestiaux ${ }^{3}$. Cette machine, toute simple, se compose d'une grosse chaudière alimentée au fuel, au charbon ou au bois, qui envoie par des tuyaux de la vapeur dans des paniers métalliques fermés contenant les pommes de terre. Deux paniers sont disposés sur une plate-forme mobile à côté de la chaudière : l'un se fait remplir pendant que l'autre est utilisé pour la cuisson. À SaintBrice, la chaudière mesure 4 mètres de long pour 1,2 mètre de diamètre et elle permet de cuire en une demi-heure $300 \mathrm{~kg}$ de pommes de terre. Tirée par un tracteur, elle passe de ferme en ferme et, en 3 jours, un groupe de 4 agriculteurs cuit et tasse dans des silos tapissés de papier goudron environ 15 à 20 tonnes de tubercules, c'est-à-dire la production annuelle moyenne d'une exploitation. Il s'agit d'un ensilage identique à celui des betteraves, sauf que pour les pommes de terre il faut le faire précéder d'une cuisson pour éliminer les germes et empêcher le gel.

L'étuveuse, je la connais - sans l'avoir vue toutefois - par les propos d'agriculteurs en retraite de Saint-Brice. Ces entretiens ont été réalisés dans le cadre d'une recherche plus large qui porte sur le rôle des associations d'agriculteurs et de la coopération dans le développement agricole aux XIX et $x^{\mathrm{e}}$ siècles. En 1953, cette machine est présentée par Monsieur Gellier, conseiller agricole du canton de Saint-Brice-en-Coglès, comme indispensable au développement des fermes de l'est du département qui, en complément à l'élevage laitier, commencent alors à intensifier l'élevage du porc. La proposition apparaît particulièrement alléchante : certes, la machine coûte cher (900000 francs), mais l'achat, s'il est réalisé dans le cadre d'une CuMA, peut en être financé pour moitié par des subventions d'État. L'interlocuteur du conseiller agricole est alors Pierre Bellé, jeune agriculteur de 30 ans, qui exploite en fermage une terre de 10 hectares, et qui a fait un peu de vulgarisation agricole dans le cadre de la JAC. Soutenu par 3 ou 4 camarades " qui s'entraident " pour les gros travaux agricoles, il décide alors de créer une CuMA, en sollicitant par porte-à-porte une soixantaine d'adhérents domiciliés sur 3 cantons. L'investissement

remède à ce gaspillage a été trouvé dans la cuisson des tubercules par étuvage, suivie d'une conservation par ensilage. La cuisson stérilise la masse et la met à l'abri de toute fermentation. La richesse de la pomme de terre en amidon favorise la fermentation lactique qui dispense d'avoir recours à des agents de conservation. La cuisson s'effectue par étuvage à basse pression.

3. Lors de la journée internationale de matériel pour la culture de pomme de terre en 1949, l'école de Grignon présente cette machine appelée " cuiseuse ". Différents modèles qui fonctionnent au gaz mais aussi à l'électricité sont exposés, La pomme de terre, septembre $1949, \mathrm{n}^{\circ} 121$, p. 22. 
demandé à chaque cumiste est proportionnel à la superficie qu'il exploite mais demeure modique (le capital initial de la CuMA est fixé à 240000 francs divisé en 240 parts de 1000 francs, le versement le plus élevé est de 7000 francs), une partie de l'achat étant financée par un emprunt. L'étuveuse est achetée et utilisée dès 1955; un employé à mi-temps rétribué par la CumA, s'occupe de la machine, le transport de cette dernière reposant sur l'entraide entre agriculteurs : ceux qui possèdent un tracteur assurent la corvée pour le voisinage. L'étuveuse sera utilisée jusqu'en 1970; son abandon correspond alors à la victoire du maïs sur la pomme de terre ainsi qu'à la mise en place des grands élevages porcins nourris aux " aliments".

\section{Une étuveuse à pommes de terre}

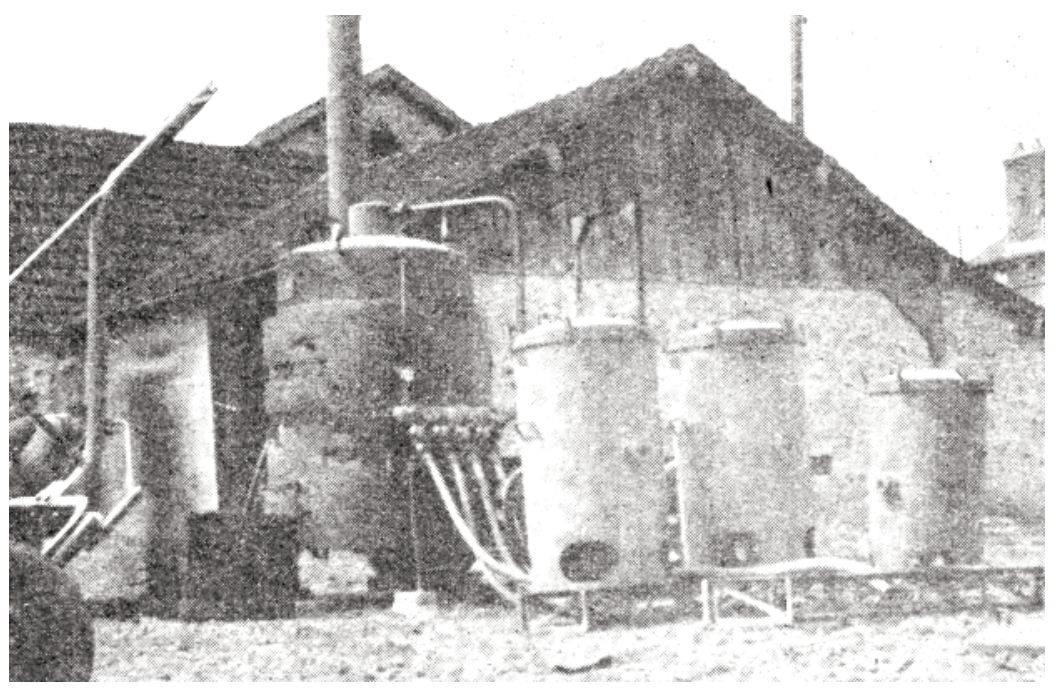

Source - La pomme de terre, $\mathrm{n}^{\circ}$ 121, septembre 1949.

\section{Une adoption étonnamment facile}

Jusqu'à la veille de la guerre, l'agriculture se fait dans le cadre de petites exploitations (68\% ont moins de 10 hectares en 1929) qui sont gérées à part égale en faire-valoir-direct et en fermage. Pratiquant le système polyculture-élevage (céréales/vaches laitières) elles utilisent pour l'essentiel une main d'œuvre familiale (27 fermes sur 190 emploient des salariés). 
Structure des exploitations de Saint-Brice-en-Coglés en $1929^{4}$

\begin{tabular}{|c|c|c|c|c|c|c|c|}
\hline Superficie & $<3$ ha & $3-5$ ha & $5-10$ ha & $10-20$ ha & $20-30$ ha & $30-40$ ha & Ensemble \\
\hline $\begin{array}{c}\text { Nombre et } \\
\text { pourcentage } \\
\text { d'exploi- } \\
\text { tations }\end{array}$ & 34 & 37 & 58 & 52 & 6 & 3 & 190 \\
\hline
\end{tabular}

Ces exploitations sont faiblement mécanisées puisqu'à cette date on ne compte que 13 tracteurs et 3 motoculteurs dans la région de Fougères; les moissonneuses-batteuses, les arracheuses de pomme de terre, les semoirs, les distributeurs d'engrais que l'on trouve ailleurs dans le département qui est le mieux équipé de la Bretagne - sont absents à Saint-Brice-enCoglès 5 .

Ce n'est donc pas surprenant si l'étuveuse, machine peu diffusée dans l'Ouest, est inconnue de tous lorsque le conseiller agricole l'évoque; seuls quelques anciens prisonniers de guerre l'ont vu fonctionner en Allemagne. Or, l'effort financier nécessaire pour former la CuMA, n'a, selon la mémoire des administrateurs, posé aucun problème. Une fois les agriculteurs sollicités, l'argent est arrivé très vite, et aucune opposition ne s'est manifestée. Plusieurs paramètres ont joué un rôle dans cette conversion rapide du Coglès au progrès technique.

\section{L'évolution du système de production}

L'étuveuse est en phase avec les transformations agricoles de l'aprèsguerre, entre autre avec le début de l'intensification de l'élevage porcin. En Bretagne, l'effectif porcin connaît une croissance continue depuis le milieu du XIX ${ }^{\mathrm{e}}$ siècle, et ce mouvement s'accélère à partir de 1945 puisque le nombre de porcs se trouve multiplié par deux entre 1938 (467000 têtes) et 1960 (1 161000 têtes $)^{6}$. Après la guerre, la spécialisation porcine n'est pas encore affirmée mais les fermes bretonnes commencent à élever plus de cochons : on en comptait en moyenne 2 par ferme à la fin du XIX ${ }^{\mathrm{e}}$ siècle, ils sont près de 6 en 1955. Dans le canton de Saint-Brice, le nombre de têtes tarde à s'accroître : le recensement agricole de 1929 ne fait état que de 1729 cochons pour 1716 fermes; celui de 1955 se fait l'écho d'une réelle progression - inférieure cependant à la moyenne bretonne - puisqu'il dénombre 4491 têtes soit 2,6 cochons par exploitation. Toutefois, dès le milieu des années cinquante, quelques exploitations élèvent une dizaine

4. Enquête agricole de 1929, Arch. dép. d'Ille-et-Vilaine, 6M984.

5. Répartition des tracteurs et des motoculteurs dans les exploitations en 1946, Arch. dép. d'Ille-et-Vilaine, W1382.

6. L'augmentation de la production porcine bretonne qui a été relativement tardive puisqu'elle démarre après 1890, apparaît ensuite exceptionnelle car à l'échelon national, le nombre de têtes n'a quasiment pas augmenté entre 1890 (7421000 porcs) et 1960 (8063000), P. Daucé et Y. Léon, L'évolution de l'agriculture bretonne depuis 1850, les systèmes de culture et d'élevage, fascicule 3, INRA, 1982. 
de porcs, mais ce n'est que vers 1970 que seront créées quelques grandes porcheries abritant plus de 100 animaux. Jusqu'alors, l'augmentation du cheptel n'exigeait pas une transformation des modes d'élevage : les cochons sont toujours nourris de façon traditionnelle par les femmes qui font cuire dans le petit lait provenant de la fabrication du beurre fermier, les pommes de terre dont la culture demeure encore considérable en Bretagne (8\% de la SAU entre 1830 et 1860 - 4 à $5 \%$ sur le reste du territoire national). Ce n'est que plus tard, vers 1970, que les pommes de terre ne suffiront plus pour nourrir la centaine de cochons ${ }^{7}$ des porcheries; il faudra alors recourir aux " aliments". Ces années correspondront d'ailleurs à la régression de la culture du tubercule ainsi qu'à l'arrêt de la fabrication du beurre fermier et à l'abandon de l'étuveuse. Cette dernière a donc été donc un objet technique qui répondait à la mise en route de la spécialisation porcine.

\section{La curiosité technologique des jeunes agriculteurs}

Les promoteurs de l'étuveuse, qui ont aujourd'hui plus de soixante-dix ans, évoquent la fascination qu'ils éprouvaient pour la technique dans les années cinquante. Leur formation de base était légère, la plupart avaient arrêté leurs études après le certificat d'études et n'avaient pas reçu de formation agricole ${ }^{8}$ : seuls trois jeunes gens du canton étaient allés à l'école d'agriculture des Trois-Croix, six autres, dont Pierre Bellé, avaient suivi les cours agricoles par correspondance, deux étaient abonnés aux cours d'agriculture par correspondance, et quelques-uns avaient participés à des journées de formation organisées par les responsables locaux de la JAC. Ils évoquent encore avec plaisir ces enseignements où " on découvrait tout". Toutefois, les propos tenus par ces anciens cumistes mettent en évidence d'autres influences que l'enseignement : la guerre semble avoir joué, un rôle essentiel. D'une part, les quatre années de conflit vont diffuser de nouvelles techniques et mettre en évidence la puissance qu'elles procurent, d'autre part, elles vont ouvrir l'horizon de nombreux agriculteurs. Parmi les cumistes de la première heure, les prisonniers de guerre représentent plus de la moitié de l'effectif, et leur dynamisme est souvent rappelé au cours des entretiens que nous avons menés. Nos interlocuteurs, qui furent prisonniers en Bavière, évoquent plusieurs aspects de ce qu'ils avaient perçu comme signes de modernité en Allemagne : le matériel, d'abord avec les botteleuses, les lieuses, les batteries, les planteuses de pommes de terre, les arracheuses et les étuveuses, voire les tracteurs; mais aussi l'organisation du travail car ils y ont découvert les coopératives de matériel,

7. À partir des années soixante-soixante-dix, l'élevage porcin se développe de façon exponentielle et l'on remarque alors un décrochement net avec les productions végétales traditionnelles : orge et pommes de terre. Ce nouveau type d'élevage correspond aux ateliers hors-sol et fonctionne de façon nouvelle par rapport au système de polyculture-élevage.

8. Recensement agricole de 1955, Arch. dép. d'llle-et-Vilaine W1382, art. 3. 
les coopératives laitières et le contrôle du lait. L'élevage aussi leur paraît d'une autre dimension : l'un d'entre eux évoque la porcherie de 150 cochons qu'il devait nourrir. Mais c'est le confort des maisons qui les a le plus impressionnés : les habitations étaient grandes, très propres, on y trouvait plusieurs chambres dont certaines pour les employés, des douches, l'eau courante, l'électricité. Certes, rien de nouveau dans tout cela : une grande partie du matériel découvert en Allemagne était présent dans les grandes fermes françaises et même bretonnes à la même époque ${ }^{9}$, mais les paysans des petites exploitations n'avaient pas eu l'occasion de le découvrir. L'habitat était particulièrement vétuste en Bretagne ${ }^{10}$ et demeurait très en retard sur les fermes du Nord de la France reconstruites après 1914 : l'eau courante n'était pas installée, toute la famille dormait souvent dans une seule chambre voire dans la pièce unique, les cours des fermes restaient boueuses toute l'année. Il est certes difficile de mesurer l'impact de ces découvertes sans mener une enquête plus approfondie ${ }^{11}$ mais de nombreux témoignages rappellent que cet épisode, si douloureux fut-il, permit à certains d'envisager une autre façon d'être agriculteur. Les anciens prisonniers évoquent d'ailleurs leur rapide déception au retour, lorsqu'ils se heurtèrent aux propriétaires qui refusaient alors de participer à la rénovation des logements.

L'étuveuse est donc apparue comme un moyen de s'approprier la modernité, doté de plusieurs atouts capables de séduire les sceptiques. D'une part, bien qu'elle soit imposante, elle s'utilise simplement et n'exige pas de grandes capacités techniques; d'autre part, elle ne remet pas en cause l'organisation du travail dans la ferme car la femme reste celle qui nourrit les porcs, sa tâche est seulement facilitée. De plus elle n'implique pas une modification des techniques de production : le menu ancestral des cochons n'est pas modifié, il repose toujours sur les produits de la ferme. En outre, l'étuveuse illustre parfaitement l'intérêt d'un service commun : son prix d'achat est élevé mais elle n'est utilisée que trois ou quatre jours par an sur une exploitation, par conséquent l'alternative à la propriété collective ne peut être que l'appel à un entrepreneur de matériel agricole, or aucun de ces derniers ne proposait alors cette machine. En 1950, l'achat d'une étuveuse se pose dans les mêmes termes que celui d'une moissonneuse dans les années 1960.

9. En 1946, une enquête rappelle que l'Ille-et-Vilaine est le département le mieux équipé de la Bretagne. On y trouve environ 350 tracteurs, 70 faucheuses, environ 200 moissonneuses (lieuses ou batteuses), 20 arracheuses de pommes de terre, Bulletin récapitulatif départemental, Arch. dép. d'Ille-et-Vilaine W1382.

10. En 1954, 77 \% des logements agricoles avaient été construits avant la guerre de 1914, $53,9 \%$ des maisons avaient une seule pièce, $28,7 \%$ disposaient de deux pièces, $50 \%$ étaient raccordées au réseau électrique, et une maison sur 300 avait l'eau courante, LEFEVRE, Denis, À l'ombre des machines, les CUMA, 50 ans de solidarités locales, Éditions Entraid', 220 p., p. 51.

11. Il faudrait probablement coupler des interviews avec une enquête statistique portant d'une part sur le nombre de prisonniers placés dans les fermes allemandes et d'autre part sur les régions de détention et l'équipement agricole que ces dernières possédaient. 


\section{La " révolution silencieuse ${ }^{12}$ "}

Lorsque dans les entretiens, les cumistes recherchent les raisons qui les ont convaincus de la nécessité d'acheter l'étuveuse, ils rappellent leur volonté de diminuer les charges qui pesaient sur les femmes, afin que celles-ci soient plus disponibles pour le foyer et l'éducation des enfants. Ces propos rappellent bien sûr ceux de la JAC et de la JACF, et ce n'est pas un hasard car, dans les années cinquante, l'Ille-et-Vilaine est un des départements où la presse jaciste est la plus lue ${ }^{13}$ et de nombreux cumistes de Saint-Brice sont d'anciens militants. La JAC, qui a fait le pari du progrès agricole, (" on ne peut être contre les machines " écrit le responsable René Colson), insiste sur la nécessité de la formation professionnelle allant jusqu'à organiser dans ce but des cours par correspondance. Dans l'est du département, les journées rurales de la JAC, comme celles qui sont organisées autour de démonstrations de tracteurs à Dourdain en 1947 sont l'occasion de montrer une nouvelle agriculture en train de naître. Mais l'Action Catholique rurale aborde aussi, dès avant la guerre, des sujets nouveaux : le statut de la femme est choisi comme thème d'année de la JAC en 1935.

La décennie 1950-1960 est la belle époque de la JAC et de la JACF, qui affirment leur volonté de prendre en charge tous les aspects de la vie des jeunes ruraux en conduisant " la révolution silencieuse des paysans ${ }^{14}$ ". Cette dernière passe entre autre par une réflexion sur la modernisation des entreprises agricoles et de l'habitat rural ainsi que sur le rôle de la femme dans l'exploitation et dans la famille. Aux environs de 1950, la JAC s'intéresse plus au rôle de la femme dans la sphère privée qu'à sa place dans la vie professionnelle et sociale : elle veut faire reconnaître la place de la femme au foyer et son rôle dans l'éducation des enfants. La femme doit certes comprendre les difficultés du mari, ne pas le freiner dans son désir d'évolution mais elle doit surtout être " maîtresse de maison, épouse et mère "; sa " libération " passant par la modernisation de l'entreprise mais aussi par celle de la maison. Les combats pour améliorer la vie privée et pour moderniser les exploitations doivent être menés de front car trop souvent, selon les JAC, les hommes épargnent pour acheter des terres, voire des machines, mais répugnent à consacrer une partie du budget à tout ce qui améliorerait le quotidien des femmes, or la technique "doit être au service de la famille... ". À l'aune de ce discours, l'étuveuse se pare d'un atout supplémentaire : ne satisfait-elle pas le goût masculin pour la technique tout en simplifiant la vie des femmes? Les femmes auraient-elles choisi cet objet

12. Titre d'un ouvrage de Michel Debatisse, La Révolution silencieuse, Calmann-Lévy, 1963, 275 p.

13. En 1954, en Bretagne plus de $5 \%$ de la population rurale de 15 à 54 ans liraient la presse de l'Action Catholique Rurale, alors que la moyenne nationale n'est que de 2,9\%, DuRUPT, Marie-Josèphe, Les mouvements d'action catholique facteur d'évolution du milieu rural, thèse de $3^{\mathrm{e}}$ cycle, université Rennes 2, s.d., 2 t., 411 et 417 p. dactyl.

14. Propos de Michel Debatisse, secrétaire général du mouvement. Cité dans : CHOLvy, Gérard, Histoire des organisations et mouvements chrétiens de jeunesse en France, Paris, Cerf, 1999, 419 p., citation p. 295. 
particulier si on leur avait demandé ce qui pouvait leur simplifier la vie? Les entretiens que nous avons menés n'évoquent jamais d'avis féminins et aucune femme n'a participé ensuite à la CuMA... L'étuveuse est un projet d'hommes.

Pour faire aboutir leurs objectifs, les mouvements de jeunesse catholique encouragent l'action collective et "l'engagement " dans la vie locale par les associations professionnelles ou politiques; René Colson, affirme que " l'organisation actuelle du monde rural rend la vie de chacun plus difficile... Dans cette société inhumaine, s'associer c'est non seulement s'en sortir mais aller vers une vie plus complète ${ }^{15}$ ". L'influence des militants sur la création et sur l'évolution des CumA est incontestable pour toute la France ${ }^{16}$ et le discours jaciste a été entendu par les jeunes agriculteurs de plusieurs cantons de l'est du département : dès 1947, à Dourdain (commune de l'est du département), une Cuma de matériel est fondée par quelques jacistes qui achètent en commun un tracteur ${ }^{17}$. Les propositions gouvernementales prévues pour aider les paysans à participer à la vie économique par le développement d'un réseau coopératif puissant - et c'est dans ce cadre que des subventions sont offertes aux CuMA qui investissent dans du matériel - tombent donc sur un terrain bien préparé.

Certes, des associations agricoles existaient bien avant que se développe l'influence de la JAC. En 1879, un comice, nettement républicain est créé à Saint-Brice, il regroupe plus de 70 membres qui s'adonnent aux activités habituelles de ce type d'organisme, et participent, par les concours surtout, à l'évolution de l'élevage qui caractérise la région à la fin du XIX siècle. Dans la première moitié du Xx $\mathrm{x}^{\mathrm{e}}$ siècle, ce sont de nombreuses mutuelles et assurances qui prennent le relais. Mais ces mouvements associatifs traditionnels ont toujours été animés par des propriétaires fonciers, dont un certain nombre appartenaient à la noblesse. C'est en opposition à cette élite locale que se situent les jeunes agriculteurs des années cinquante. Le conseiller agricole qui tente l'adoption de l'étuveuse s'adresse non pas à un propriétaire terrien mais à un jeune fermier, militant de la JAC. Celui-ci contacte trois autres agriculteurs de son âge qui ne sont pas ses plus proches voisins mais qui lui donnent " un coup de main " lors des gros travaux, et avec qui il partage une certaine communauté d'esprit. Ce groupe contacte alors, de bouche à oreille, une soixantaine d'autres agriculteurs dispersés sur 3 cantons. Une étude plus détaillée des 65 adhérents à la CuMA met en évidence des caractéristiques qui, sans être communes à tous, donnent quand même une certaine coloration au groupe : la jeunesse (la majorité des adhérents ont moins de 30 ans) bien qu'aucun conflit de génération ne soit apparu, l'adhésion à la JAC, le fait d'avoir été prisonnier de guerre, et également le statut de fermier puisque seulement 3 d'entre eux étaient propriétaires-exploitants. Mais leur unité se fit surtout par la

15. LEFEVRE, Denis, op. cit.

16. DuRUPT, Marie-Josèphe, op. cit. p. 263.

17. LEFEVRE, Denis, op. cit., p. 44. 
volonté, partagée par un grand nombre d'entre eux " de s'unir " car l'étuveuse "c'était tout un esprit " affirment nos interlocuteurs.

Ces remarques doivent être confrontées aux résultats d'autres recherches portant sur la diffusion de l'innovation en milieu rural après la seconde guerre mondiale. Selon des chercheurs américains ${ }^{18}$, les premiers à adopter les techniques nouvelles sont les agriculteurs les plus riches, les plus jeunes et ceux qui jouissent d'un statut social élevé. Maryvonne Bodiguel, dans son analyse de la diffusion technique ${ }^{19}$ (la machine à traire et le tracteur) dans les Côtes-du-Nord après la seconde guerre mondiale confirme le rôle de la jeunesse mais aussi du statut social (ce qui n'est pas la notabilité). Ces précurseurs sont influencés par des interventions extérieures au groupe (documentation d'origine diverse et démonstrations), alors que pour les autres - ceux qui reproduisent les choix des innovateurs - le moyen de diffusion le plus rapide devient " la connaissance par l'expérience " qui passe en premier par " le coup d'œil " chez le voisin dans le cadre des équipes d'entraide aux travaux agricoles. Par conséquent, les démonstrations, les foires-expositions, les publicités jouent sur ces derniers un rôle nettement plus faible - en France du moins - et la décision d'acquérir une nouvelle machine ne progresse que très lentement. Selon les chercheurs américains, la diffusion de l'innovation se fait plus précisément en cinq stades successifs : la prise de conscience de l'objet, l'intérêt, l'évaluation, l'essai, l'adoption. Dans les Côtes-du-Nord, Maryvonne Bodiguel reconnaît ces différentes étapes, qu'elle perçoit toutefois comme simultanées et non pas successives, et elle conclut, en accord avec les américains, en notant qu'un des problèmes fondamentaux du processus d'adoption est l'influence du voisinage, car, dit-elle, " le rapport entre système de valeurs et processus d'adoption est capital... L'un des problèmes essentiels de l'adoption d'une innovation est son degré de compatibilité avec le milieu dans lequel est introduite ${ }^{20}$ ".

L'acquisition de l'étuveuse ne répond pas totalement aux analyses décrites précédemment. D'une part, il s'agit d'un achat collectif, qui implique immédiatement un groupe, sans que l'on puisse observer la diffusion par contact. Mais ce qui surprend surtout, c'est la rapidité avec laquelle a été prise la décision, ainsi que le nombre de personnes concernées. Les trois jeunes initiateurs, qui sont habitués à travailler ensemble, se sont rapidement laissés convaincre par le conseiller agricole, puis, très vite, les 65 premiers engagements ont été pris "sur papier ", toujours sans photo, ni démonstration, ni essais, les trois jeunes faisant du porte-à-porte bien audelà de leur cercle de relations et de leur équipe d'entraide. Ce n'est qu'en-

18. Rogers, M., Diffusion of innovations, New-York, The free press of Glencoe, 1962 et GROSS, N., " The differencial characteristics of acceptors and non acceptors of agricultural practices ", Rural Sociology, 1949, 14.

19. Bodiguel, Maryvonne, "La diffusion des innovations techniques dans deux communes des Côtes-du-Nord ", Études rurales, 1968, 30, p. 43-86.

20. Ibidem, p. 75 . 
suite que l'influence du voisinage et de l'expérience commune interviendra peut-être, lorsqu'il faudra convaincre les derniers adhérents de la CuMA qui seront jusqu'à 125 membres dans les années 1960. Toutefois cette adoption très volontariste qui écarte tout test, toute formalité et qui touche un nombre exceptionnel d'individus nous paraît bien illustrer une part des propos de Maryvonne Bodiguel : l'étuveuse est un outil hautement compatible avec le milieu agricole breton de l'après-guerre, compatibilité économique que nous avons déjà évoquée, mais surtout compatibilité « intellectuelle ". L'acquisition de cette machine nous semble plus un engagement idéologique par rapport à l'agriculture de groupe qu'une réponse à un besoin technique ou économique, même si ces derniers ne sont pas négligeables pour quelques individus. L'étuveuse est le prétexte à une nouvelle façon de vivre l'agriculture par des jeunes qui connaissent les difficultés présentes et perçoivent les mutations futures, autrement dit, cet achat nous paraît être un prétexte à la mise en place d'une CUMA ${ }^{21}$.

Cette affaire aura-t-elle des répercussions sur la facilité d'adoption d'autres matériels? Il est difficile de répondre à cette question en l'état actuel de l'enquête. Les agriculteurs vont s'équiper souvent individuellement, parfois de façon très lente jusqu'en 1970; ils feront aussi appel aux services des entrepreneurs de matériels agricoles. Dans le cadre de la CumA l'étuveuse ne débouchera d'ailleurs pas sur un essor technologique à tout crin : seule une tronçonneuse complétera l'équipement. Toutefois, le mouvement coopératif va se développer dans le canton : les plus actifs de Saint-Brice vont fonder une autre CumA dès 1959, celle de "l'avenir Briçois " dite la "CumA des pauvres" qui existe toujours. Elle interviendra sur une zone géographique plus restreinte et proposera donc d'autres matériels. En réponse aux nouveaux besoins de l'agriculture, elle se développera autour de plusieurs axes : gros outils, congélation, banque de travail et d'entraide et actuellement prise en compte des problèmes écologiques du bocage. Mais, même si l'esprit des initiateurs, c'est-à-dire " la volonté d'être ensemble " semble actuellement toujours très présente chez les responsables des Cuma du Coglès, dès 1970, les structures collectives ne provoquent plus l'enthousiasme des débuts. Une enquête menée en 1970 sur 117 agriculteurs dévoile que seul un tiers utilise en priorité le matériel de la CumA (tout en possédant quelques machines) : ils exploitent souvent des fermes 10 à 40 hectares. Les deux tiers refusent la CUMA, ou l'utilisent accessoirement : c'est le cas de quasiment tous les petits agriculteurs (moins de 10 hectares) et de la moitié des moyens et grands exploitants (plus de 10 ha). L'enthousiasme pour l'étuveuse a bien correspondu à un moment précis de l'histoire rurale de l'Ouest.

21. Juste après la guerre, de nombreuses CuMA avaient été crées en Bretagne : en 1947, on en comptait 593, mais il n'en restera plus que 250 en 1952 et 60 en 1955. Ces CuMA souhaités par Tanguy-Prigent, n'ont souvent pas fait long feu, car plusieurs n'étaient que prétextes pour quelques agriculteurs malins qui ainsi se procuraient, à bas prix, des tracteurs importés des États-Unis. Les CumA de la seconde génération, qui seront durables reposent sur des objectifs radicalement différents, LEFEVRE, Denis, op. cit., p. 45. 


\section{RESUME}

En 1955, un groupe de jeunes agriculteurs de l'est de la Bretagne décide d'acheter en commun une étuveuse à patates. Pour cela, ils créent une des premières Cuma (Coopérative d'Achat de Matériel Agricole) d'Ille-et-Vilaine. Cet investissement collectif peut être perçu comme le symbole des importantes mutations que connaît l'agriculture bretonne : intensification de l'élevage, modernisation du matériel, mise en place de coopératives. Cet article s'interroge sur les formes qu'a pris la rénovation agricole en Bretagne après la Seconde Guerre mondiale et plus particulièrement sur les circonstances qui ont permis aux jeunes agriculteurs de mener à terme leurs projets de " travailler ensemble ".

\section{ABSTRACT}

In 1955, a group of young farmers of the east of Brittany decide to buy a pressure-cooker for potatoes jointly. That's why they create one of the first CUMA (Cooperative Purchase of Farm equipment) of Ille-et-Vilaine. This collective investment can be perceived as the symbol of the significant change which concerns agriculture of Brittany: intensification of the breeding, modernisation of the farm equipment, establishment of co-operatives. The aim of this article it's to wonder about agricultural improvement in Brittany after the Second World War, and more particularly about circumstances which has authorised the farmers to plan to "work together" 
